\title{
Single-cell transcriptome analysis of the novel coronavirus (SARS-CoV-2) associated gene $A C E 2$ expression in normal and non-obstructive azoospermia (NOA) human male testes
}

\author{
Xixi Liu ${ }^{1,2,4 \dagger}$, Yidong Chen ${ }^{1,2,4,5 \dagger}$, Wenhao Tang ${ }^{6}$, Li Zhang ${ }^{2}$, Wei Chen ${ }^{1,2,4,5}$, Zhiqiang Yan ${ }^{1,2,4,5}$, \\ Peng Yuan ${ }^{2,3,4}$, Ming Yang ${ }^{1,2,4,5}$, Siming Kong ${ }^{1,2,4,5}$, Liying Yan ${ }^{1,2,3,4^{*}}$ \& Jie Qiao ${ }^{1,2,3,4,5^{*}}$ \\ ${ }^{1}$ Beijing Advanced Innovation Center for Genomics, Department of Obstetrics and Gynecology, Third Hospital, College of Life Sciences, \\ Peking University, Beijing 100871, China; \\ ${ }^{2}$ Center for Reproductive Medicine, Department of Obstetrics and Gynecology, Peking University Third Hospital, Beijing Key Laboratory of \\ Reproductive Endocrinology and Assisted Reproductive Technology, Beijing 100191, China; \\ ${ }^{3}$ National Clinical Research Center for Obstetrics and Gynecology, Beijing 100191, China, \\ ${ }^{4}$ Key Laboratory of Assisted Reproduction (Peking University), Ministry of Education, Beijing 100191, China; \\ ${ }^{5}$ Peking-Tsinghua Center for Life Sciences, Academy for Advanced Interdisciplinary Studies, Peking University, Beijing 100871, China; \\ ${ }^{6}$ Department of Urology, Peking University Third Hospital, Beijing 100191, China
}

Received March 31, 2020; accepted April 21, 2020; published online April 30, 2020

\begin{abstract}
Being infected by SARS-CoV-2 may cause damage to multiple organs in patients, such as the lung, liver and heart. Angiotensin-converting enzyme 2 (ACE2), reported as a SARS-CoV-2 receptor, is also expressed in human male testes. This suggests a potential risk in human male reproductive system. However, the characteristics of ACE2-positive cells and the expression of other SARS-CoV-2 process-related genes are still worthy of further investigation. Here, we performed singlecell RNA seq (scRNA-seq) analysis on 853 male embryo primordial germ cells (PGCs) and 2,854 normal testis cells to assess the effects of the SARS-CoV-2 virus on the male reproductive system from embryonic stage to adulthood. We also collected and constructed the scRNA-seq library on 228 Sertoli cells from three non-obstructive azoospermia (NOA) patients to assess the effects at disease state. We found that $A C E 2$ expressing cells existed in almost all testis cell types and Sertoli cells had highest expression level and positive cells ratio. Moreover, $A C E 2$ was also expressed in human male PGCs. In adulthood, the level of $A C E 2$ expression decreased with the increase of age. We also found that $A C E 2$ positive cells had high expressions of stress response and immune activation-related genes. Interestingly, some potential SARS-CoV-2 process-related genes such as TMPRSS2, BSG, CTSL and CTSB had different expression patterns in the same cell type. Furthermore, ACE2 expression level in NOA donors' Sertoli cells was significantly decreased. Our work would help to assess the risk of SARS-CoV-2 infection in the male reproductive system.
\end{abstract}

SARS-CoV-2, COVID-19, testis cells, ACE2, TMPRSS2, fetal, adult, normal, NOA

Citation: $\quad$ Liu, X., Chen, Y., Tang, W., Zhang, L., Chen, W., Yan, Z., Yuan, P., Yang, M., Kong, S., Yan, L., et al. (2020). Single-cell transcriptome analysis of the novel coronavirus (SARS-CoV-2) associated gene ACE2 expression in normal and non-obstructive azoospermia (NOA) human male testes. Sci China Life Sci 63, 1006-1015. https://doi.org/10.1007/s11427-020-1705-0

†Contributed equally to this work

*Corresponding authors (Jie Qiao (lead contact), email: jie.qiao@263.net; Liying Yan, email: yanliyingkind@aliyun.com) 


\section{INTRODUCTION}

Since COVID-19, caused by SARS-CoV-2, was discovered in December 2019, more than 2,400,000 people have been infected and over 165,000 people died worldwide as of April 20, 2020. SARS-CoV-2 as a subgenus Sarbecovirus of the genus Betacoronavirus showed similar receptor-binding domain structure with SARS-CoV, and it may use ACE2 as a receptor (Lu et al., 2020).

$A C E 2$ has been reported to be expressed in several systems such as the respiratory system, digestive system, cardiovascular system and urinary system (Zou et al., 2020). In normal mice, there is no gender difference in $A C E 2$ activity between the heart and lungs, but $A C E 2$ activity in male kidneys is higher than that in female kidneys (Liu et al., 2010). The difference may be due to the secretion of $\mathrm{E} 2$ in females (Liu et al., 2010). This suggests that ACE2 may have different expression patterns in male and female gonads. The expression of $A C E 2$ in testis cells at the single cell level has been found mainly concentrated in spermatogonia, Sertoli cells and Leydig cells (Shen, 2020; Wang and Xu, 2020). The ratio of $A C E 2$ expressing spermatogonia to all spermatogonia is similar to that in type II alveolar epithelial cells (AT2) and these $A C E 2$ expressing cells are enriched in viral reproduction pathway (Wang and $\mathrm{Xu}, 2020$ ). ACE2 has also been reported selectively expressed by adult Leydig cells and may play a crucial role in regulating steroidogenesis (Douglas et al., 2004). In NOA patients, the mRNA expression levels of $A C E 2$ and $M A S$ are decreased, suggesting that these genes may be associated with male infertility (Reis et al., 2010). In addition, previous studies have shown that TMPRSS2 (Hoffmann et al., 2020), CD147 (Wang et al., 2020), and CatB/L (Hoffmann et al., 2020) are also important for SARS-CoV-2 virus to invade host cells.

To further understand the expression pattern and function of ACE2 positive cells between normal and NOA human male testes as well as the influence of COVID-19 on male reproductive system, we used almost 4,000 spermatogenesis single cells for in-depth analysis from multiple aspects and dimensions.

\section{RESULTS}

\section{The expression pattern of $A C E 2$ in all stages of spermatogenic cells and testis somatic cells}

We used published scRNA-seq data, including seven obstructive azoospermia (OA) donors and two normal donors in a total of 2,854 single cells, to analyze $A C E 2$ expression pattern in human male testis cells (Wang et al., 2018). We identified the cell types based on the known testis cell markers and divided all cells into 14 clusters of spermatogenic cells and three types of testis somatic cells (Figure 1A,
Figure $\mathrm{S} 1$ in Supporting Information). ACE2 expressing cells could be found in almost all cell types except two types of spermatid cells (Figure 1B).

Subsequently, we calculated the percentage of ACE2 expressing cells in each cluster. Previous study used the ratio of $A C E 2$ expressing AT2 cells in all AT2 cells $(1.40 \% \pm 0.4 \%)$ as a reference to assess the risk of COVID-19 to different cell types (Wang and $\mathrm{Xu}, 2020$ ). Based on this ratio we found that spermatogonial stem cells (SSC), differentiating spermatogonia (Diff.ing SPG), leptotene spermatocytes type1 (L1), leptotene spermatocytes type 3 (L3), zygotene spermatocytes (Z), diplotene spermatocytes (D), spermatocyte 7 (SPC7), spermatids stage 1 (S1) and three types of testis somatic cells might be susceptible for SARS-CoV-2 infection. Notably, more than $90 \%$ Sertoli cells expressed $A C E 2$ (Figure 1D). In all $A C E 2$ expressing cells, Sertoli cells accounted for the largest proportion (Figure 1C). This suggested that the testis somatic cells, especially Sertoli cells, might be more susceptible than germ cells for SARS-CoV-2 infection $\left(P<2.22 \times 10^{-16}\right)$.

To analyze whether the age can influence $A C E 2$ expression in human male testes, we compared $A C E 2$ positive cells ratio and expression level between these two normal donors of different age (30 and 60, respectively) and human male PGCs. From our data we found that $A C E 2$ positive cells ratio and expression level were higher in the 30-year-old normal donor than those in the 60 -year-old normal donor $(P=0.013)$. $A C E 2$ was also expressed in human male PGCs, and the average expression level in PGCs was lower than that in the 30 -year-old donor $(P=0.022)$ (Figure $1 \mathrm{E})$. This suggested that $A C E 2$ expression level might be associated with age.

\section{Different $A C E$ 2-expression-level cells in each cell type}

Though some SSC and Sertoli cells (ST) were ACE2-positive cells, $A C E 2$ expression levels were strikingly different. To better understand the characteristics of $A C E 2$-unequal cells, we divided $A C E 2$ expressing cells into five classes based on $A C E 2$ expression level (Class A $(0,20]$, Class B $(20,100]$, Class C $(100,150]$, Class D $(150,300]$, Class E $(300,1200])$ (Figure 2A). We found that although some spermatogenic cells expressed $A C E 2$, the level of $A C E 2$ expression was low, and some cells in testis somatic cells had a relatively higher $A C E 2$ expression level (Figure 2B). Therefore, we next focused on the top $A C E 2$ expressing cell cluster, testis Sertoli cells, and found that there were more than $75 \%$ Class $E$ in Sertoli cells, higher than that in the other testis cell types $\left(P=1.2 \times 10^{-15}\right)$. This phenomenon suggested that Sertoli cells might be more vulnerable to SARS-CoV-2 infection.

We performed gene ontology (GO) analysis to investigate the gene functions of different $A C E 2$ expressing classes. From up-regulated genes, disease related signal pathways 

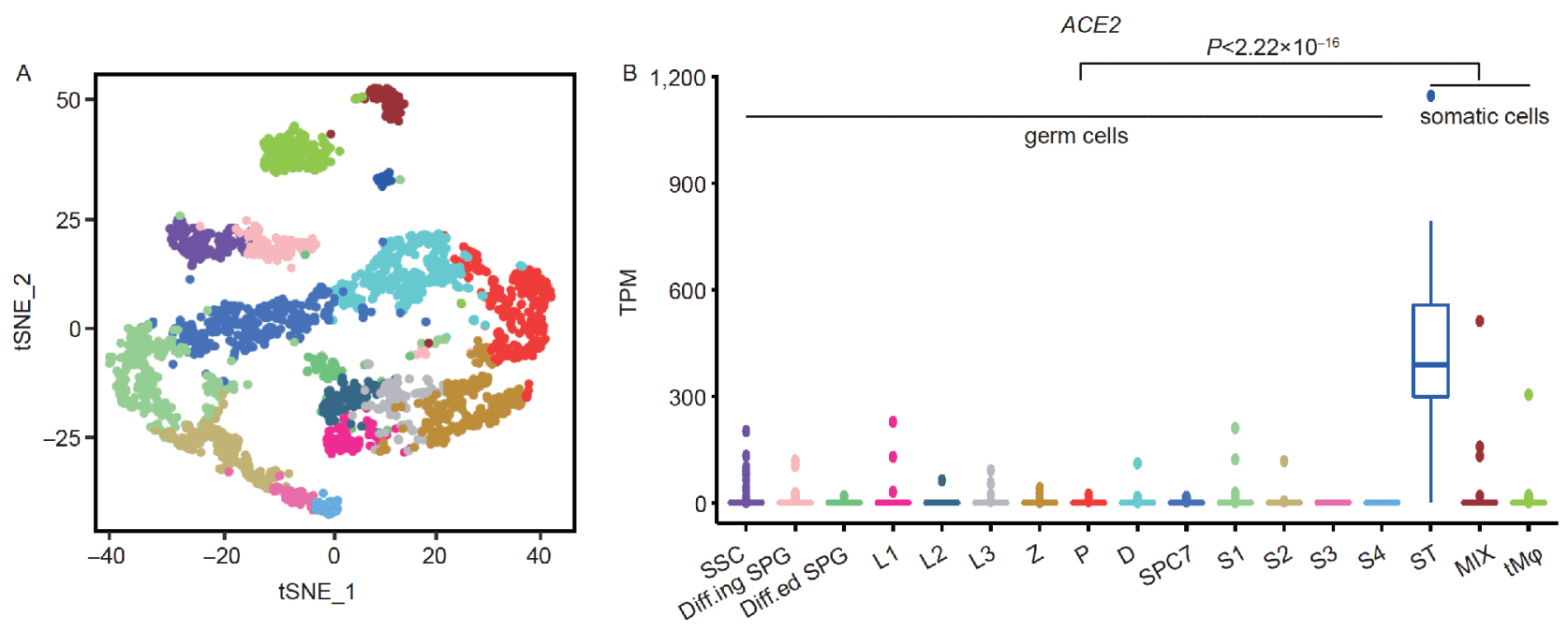

$\mathrm{C}$

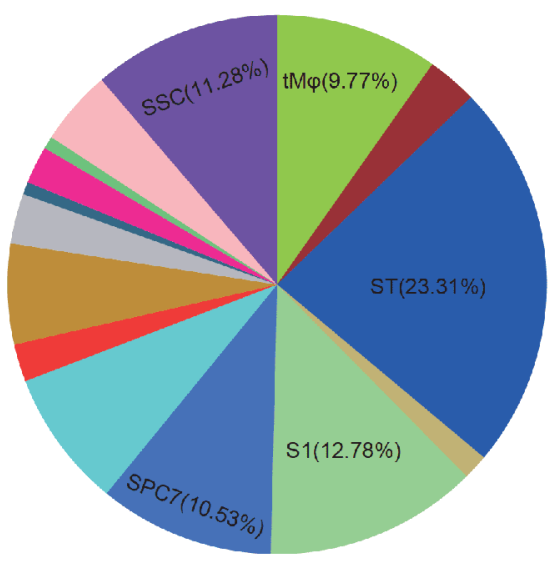

D

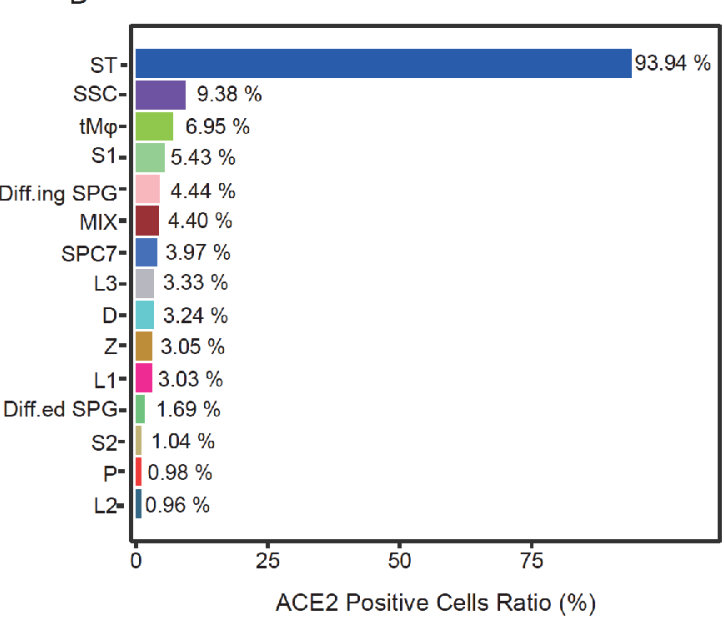

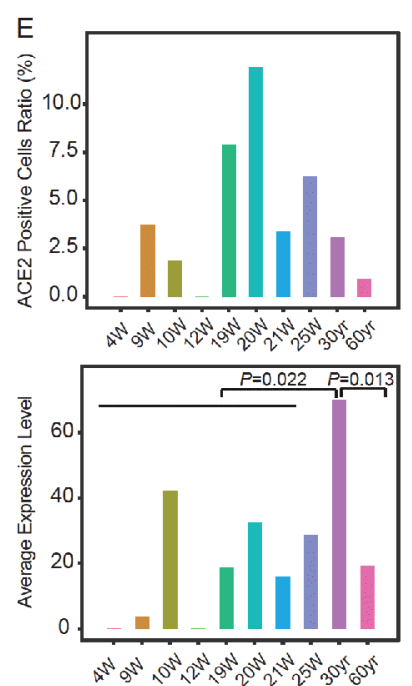

z

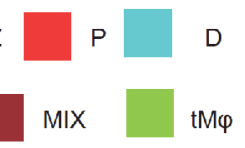

Figure $1 A C E 2$ expression pattern and the ratio of $A C E 2$-positive cells in normal testis cells. A, T-distributed stochastic neighbor embedding (t-SNE) plot of all normal 2,854 single cells in the previous paper. Different colors represent different cell types. B, The expression levels (TPM) of $A C E 2$ in all cell types. $\mathrm{C}$, Cell type distribution ratio of $A C E 2$-positive cells. Different colors represent different cell types. D, $A C E 2$-positive cells ratio in each cell type. E, $A C E 2$ positive cells ratio and $A C E 2$ average expression levels (TPM) in different age groups.

were enriched such as "viral gene expression" and "infectious disease" in Class B, "infectious disease", "viral mRNA translation" and "neutrophil mediated immunity" in Class C, "viral gene expression", "viral mRNA translation" and "platelet activation, signaling and aggregation" in Class $\mathrm{D}$, and "viral mRNA translation", "response to wounding" and "autophagy" in Class E. It showed that $A C E 2$ expressing classes were not only related to viral infection but also immune response (Figure 2C).

To further investigate the function of $A C E 2$ in male testes, we analyzed the common up-regulated genes. In all these highly expressed genes, there were only 28 genes commonly up-regulated in all four classes (Figure 2D). Then we focused on the functions of these genes which were highly expressed in all four classes. There were 16 genes related to "cellular responses to stress", and others were related to "adhesion of symbiont to host", "leukocyte activation involved in immune response", "ROS metabolic process", "homeostasis" and "cell growth arrest" (Figure 2E). Genes with the same expression trend as that of $A C E 2$ were identified with "vasoconstriction" and "regulation of reproductive process" functions (Figure 2F). These indicated that $A C E 2$ function in human male testes may be related to immune and stress response.

Then we analyzed $A C E 2$-associated network genes (gene list downloaded from genecards.org) expression level in six 


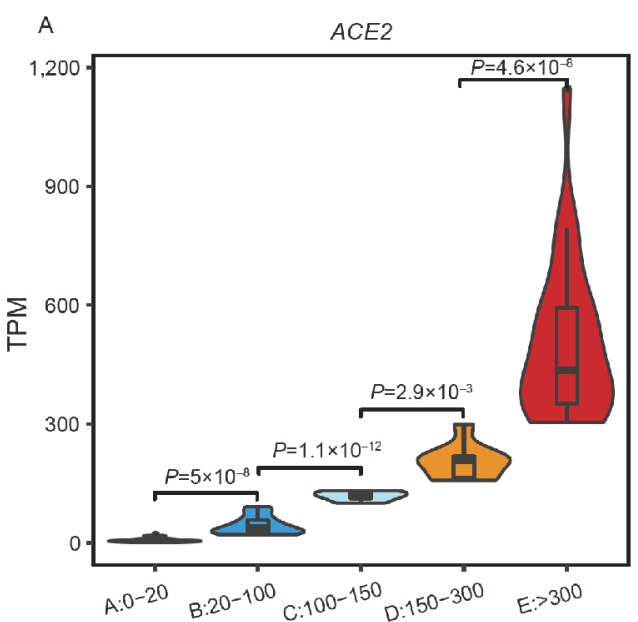

C

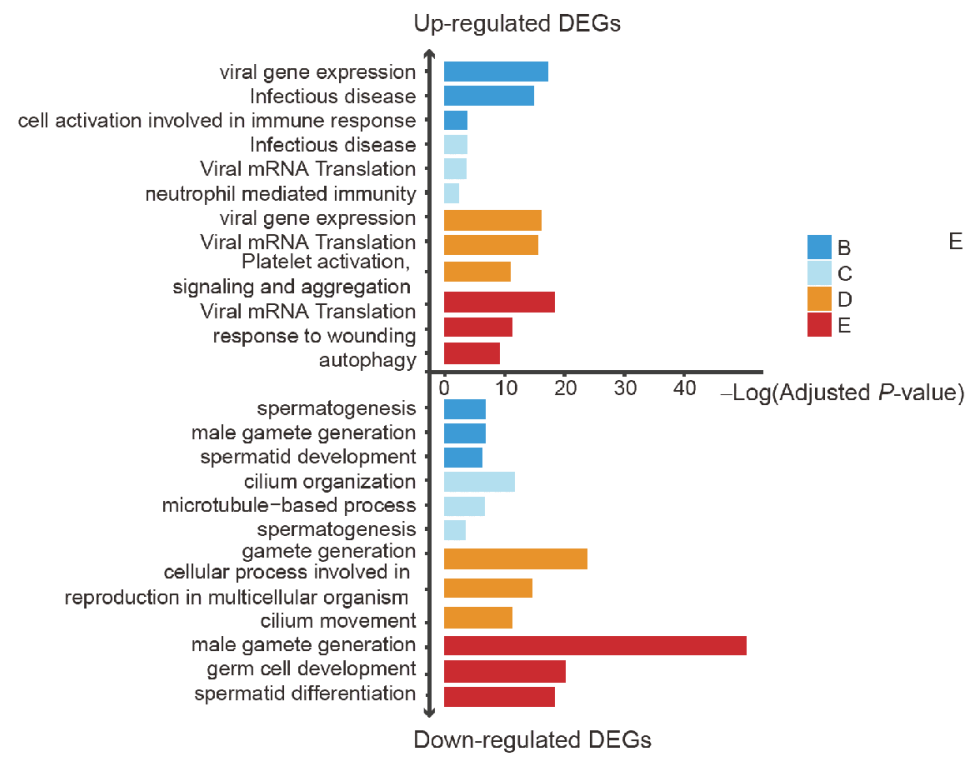

D

E
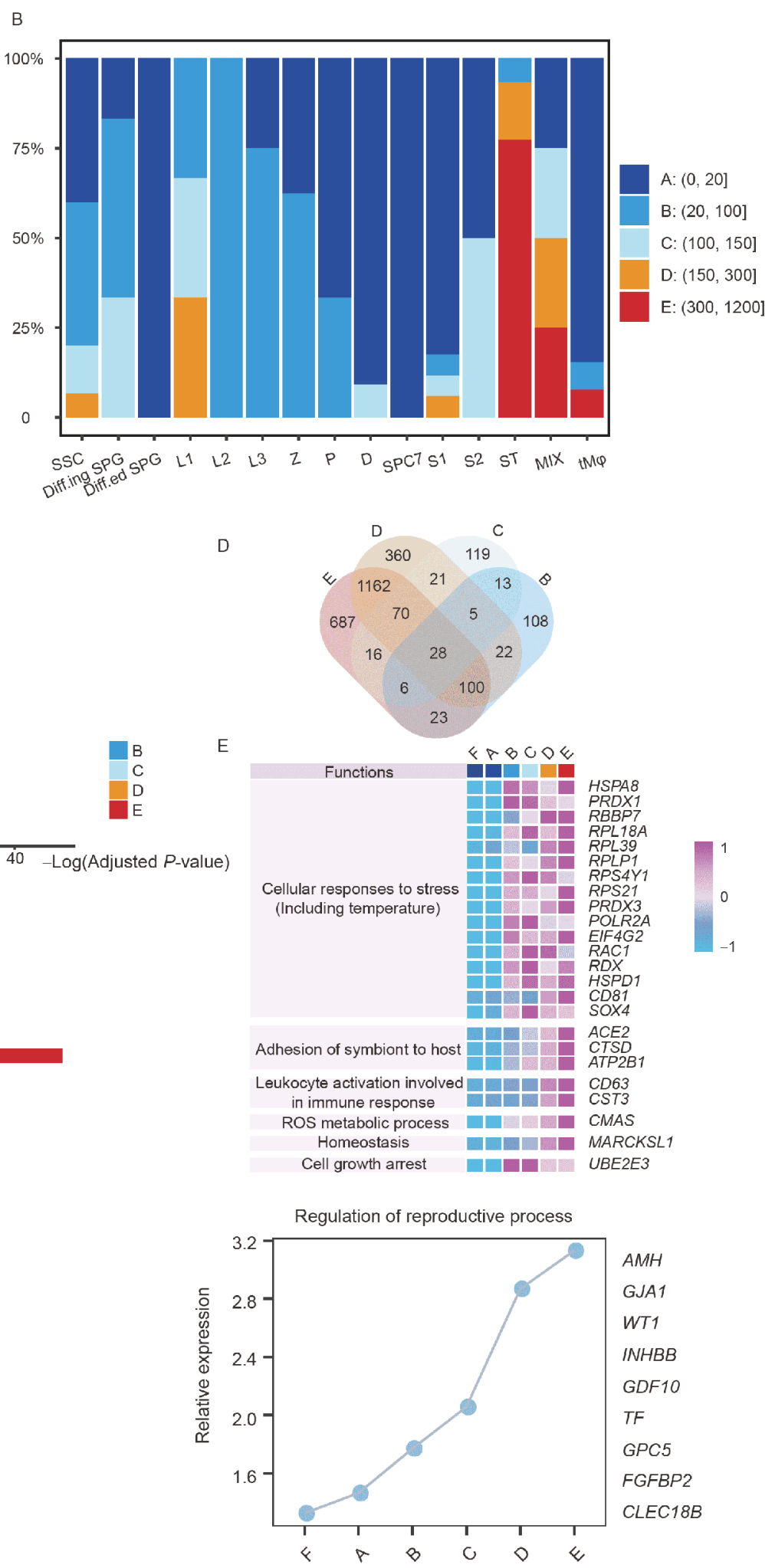

AMH

GJA1

WT1

INHBB

GDF10

TF

GPC5

FGFBP2

CLEC18B

Figure 2 Different $A C E 2$ expression levels in normal human male testis cells. A, Violin plot of five classes with different $A C E 2$ expression levels. B, Five classes composition distribution in all $A C E 2$-positive cell types. C, Gene ontology terms of different classes. D, Venn plot of intersection genes in different classes. E, Heatmap of intersection genes in all classes. Class F represents $A C E 2$-negative cells; Class A represents cells with $A C E 2$ expression in the range of 0 to 20; Class B represents cells with $A C E 2$ expression in the range of 20 to 100; Class C represents cells with $A C E 2$ expression in the range of 100 to 150 ; Class D represents cells with $A C E 2$ expression in the range of 150 to 300; Class E represents cells with $A C E 2$ expression levels greater than 300 . F, Lineplots of increasing genes. Class $\mathrm{F}$ represents $A C E 2$-negative cells; Class A represents cells with $A C E 2$ expression in the range of 0 to 20; Class $\mathrm{B}$ represents cells with $A C E 2$ expression in the range of 20 to 100 ; Class $\mathrm{C}$ represents cells with $A C E 2$ expression in the range of 100 to 150 ; Class D represents cells with $A C E 2$ expression in the range of 150 to 300; Class E represents cells with ACE2 expression levels greater than 300. 
classes (Figure S2A in Supporting Information). CAT, CHRNA10, AAMP, CHRNB1, HTR3C, ISYNA1 and CALM1 had high expression levels in Class E. Interestingly, we found $A A M P$ was associated with non-small cell lung cancer (Backhaus et al., 2017) and ISYNA1 was closely associated with lung squamous cell carcinoma (Koguchi et al., 2016). Then we analyzed the $A C E$ inhibitor pathway genes expression level. REN and ATP6AP2 were highly expressed in Class E (Figure S2B in Supporting Information). These two genes were related to mesangial cells around blood vessels in kidneys (Williamson et al., 2017). These $A C E 2$ associated genes had different expression pattern when $A C E 2$ expression changes.

From the GO term analysis in differentially expressed genes (DEGs) in different classes of Sertoli cells from normal data (Figure S2C in Supporting Information), we found some immune and virus-related pathways were enriched in up-regulated Class D and E, such as "host interactions of HIV factors" in Class D and "myeloid leukocyte mediated immunity" in Class E.

From differentially expressed genes in F, B, D and E classes of Sertoli cells, we found some genes had similar expression trend to that of $A C E 2$ (Figure S2D in Supporting Information). $C H G A$ can be a sensitive biomarker for nonsmall cell lung cancer (Qi et al., 2015) and GJA1 was also associated with human lung cancer (Zuo et al., 2017). It suggests that these genes may be correlated with $A C E 2$.

\section{Possible SARS-CoV-2 virus process-related genes expression in testis cells}

Previous study has shown that TMPRSS2 plays a significant role in SARS-CoV-2 infecting host cells (Hoffmann et al., 2020). Therefore, we next analyzed TMPRSS 2 expression in testis cells. From our data we found that almost all the cell types had TMPRSS2 expressing cells except $\mathrm{S} 4$ and $\mathrm{tM} \varphi$ groups (Figure 3A).

Interestingly, we found that the expression patterns of TMPRSS2 and ACE2 were different. In SSC cells, TMPRSS2 had the highest expression level and low $A C E 2$ expression level, while Sertoli cells had the highest $A C E 2$ expression level and low TMPRSS2 expression level (Figure 3B). The invasion of SARS-CoV-2 virus into host cells requires the combined action of TMPRSS2 and ACE2. This suggests that if the virus can invade testicular tissue, there may be interactions between testis somatic cells and spermatogenic cells.

$\mathrm{S}$ protein priming in cell lines was also associated with endosomal cysteine proteases cathepsin B and L (CatB/L) (Simmons et al., 2005), so we next analyzed the expression of CTSL and CTSB. The expression of CTSL was highest in Z stage spermatocytes, but low in SPC7 and four spermatid stages. $C T S B$ had a high expression level in testis somatic cells especially in testicular macrophages (tM $\varphi$ ) (Figure $3 \mathrm{C})$.
A recent study proposes that CD147, another possible SARS-CoV-2 viral invasion pathway, can bind to the S protein to mediate viral invasion (Wang et al., 2020). Therefore, we next analyzed the $B S G$ (CD147) expression level in testis cells. $B S G$ was expressed in all types of testis cells and had the highest expression level in Diff.ing SPG cells (Figure 3D). The results suggest that if the SARS-CoV2 virus could infect human male testes, it may enter the host cell in a variety of ways.

\section{ACE2 expression pattern in NOA patients}

$A C E 2$ has been reported to be related to human male infertility (Reis et al., 2010). Therefore, we next analyzed $A C E 2$ expression level in one Sertoli cell only syndrome (SCOS) patient (used published data) and two NOA patients with spermatogenic cells but no mature sperms.

We first used classical Sertoli cell markers such as $A M H$, $W T 1$ and $S O X 9$ to identify and select Sertoli cells from three NOA patients and normal donors (Figure S3B in Supporting Information). Then we compared $A C E 2$-positive cells ratio and $A C E 2$ expression level between them. The ratio of $A C E 2$-positive cells was similar in SCOS and normal donors but was decreased in NOA2 and NOA3 patients (Figure 4A). The Class E's ratio in normal Sertoli cells was higher than others (Figure 4B). Compared with normal and three NOA donors, we found that $A C E 2$ expression level was significantly decreased in NOA donors' Sertoli cells but the TMPRSS2 expression level showed no significant difference (Figure 4C and D).

Similar to total testis cells, the up-regulated genes GO analysis in NOA D and E classes also indicated an enrichment in immune and virus-related pathways such as "cell activation involved in immune response" and "receptormediated virion attachment to host cells" (Figure 4E). Compared with normal Sertoli cells, up-regulated genes in NOA D and E classes were enriched in virus-related pathways such as "viral transcription" and "viral mRNA Translation", down-regulated genes were enriched in human male reproduction (Figure 4F).

Then we analyzed the stress response, immune activation and spermatogenesis related gene lists expression level in normal and NOA Sertoli cells F and E classes. Both Sertoli cells in normal and NOA Class $\mathrm{E}$ had increased expression level of genes related to stress response and immune activation (Figure 4G). The spermatogenesis-related genes were significantly decreased in NOA Sertoli cells (Figure $4 \mathrm{H})$.

\section{DISCUSSION}

In our study, we found more cell types had $A C E 2$ expressing 
A

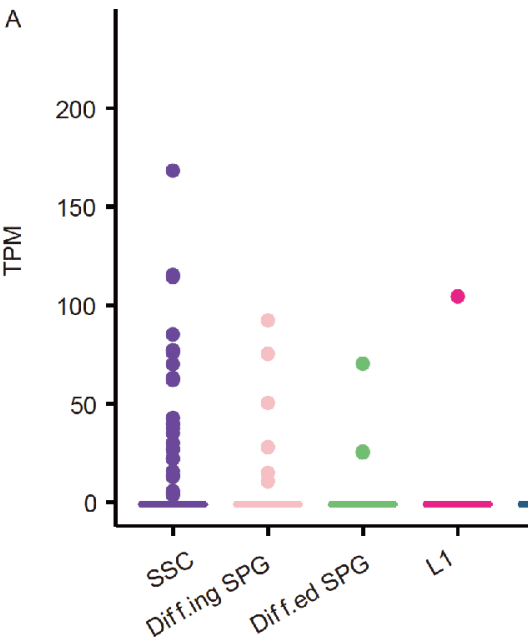

B
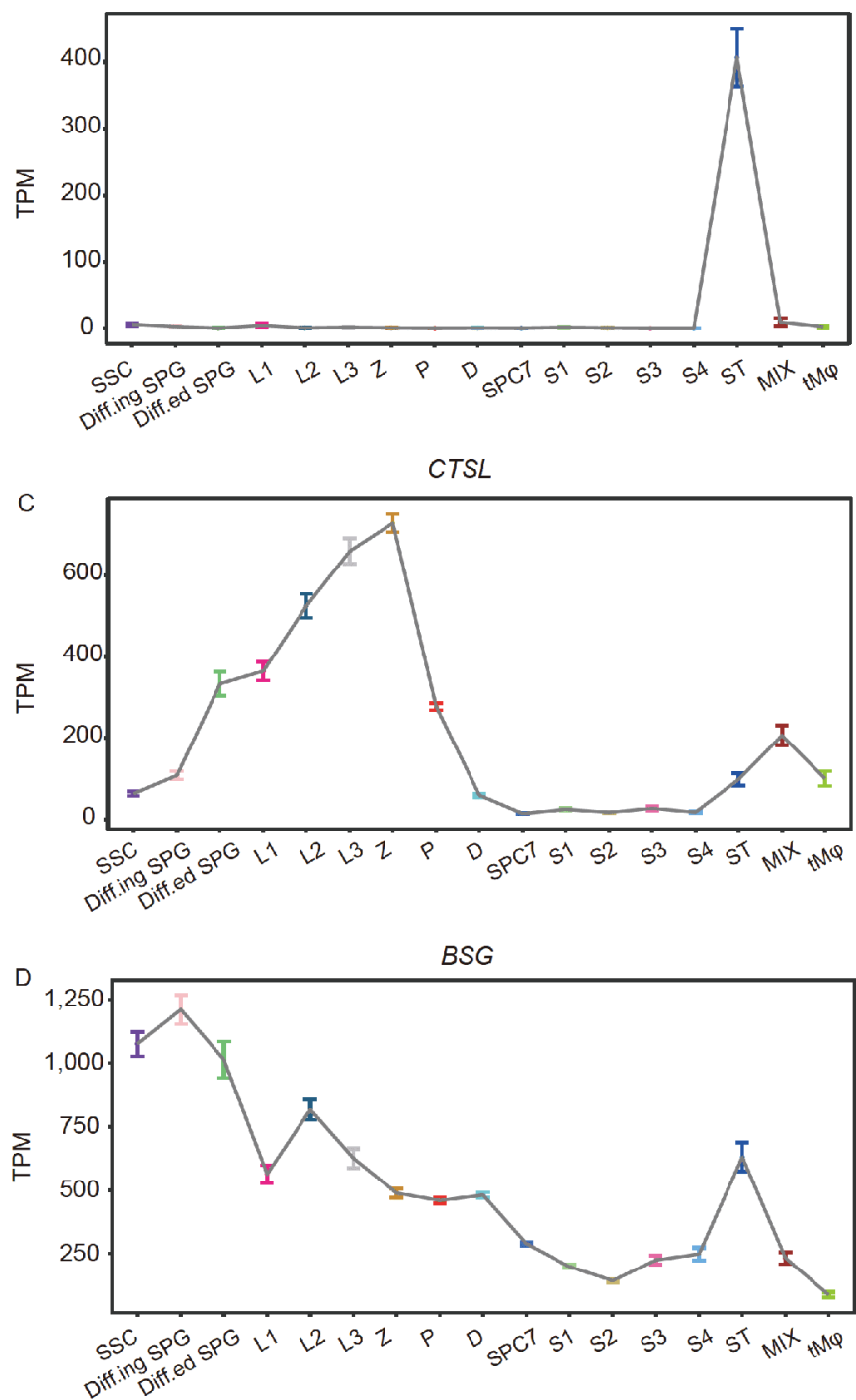

TMPRSS2

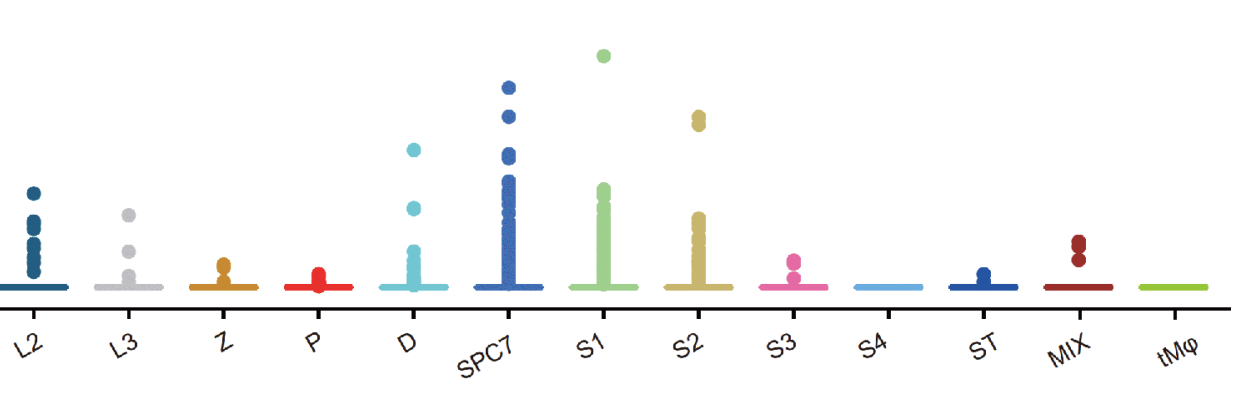

TMPRSS2

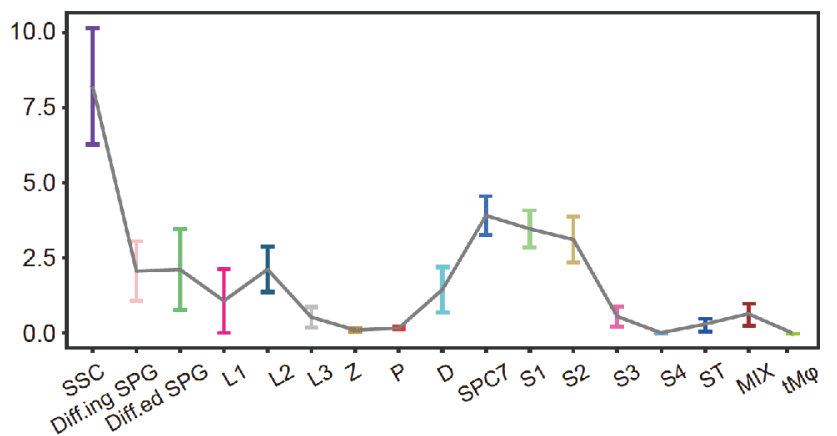

CTSB

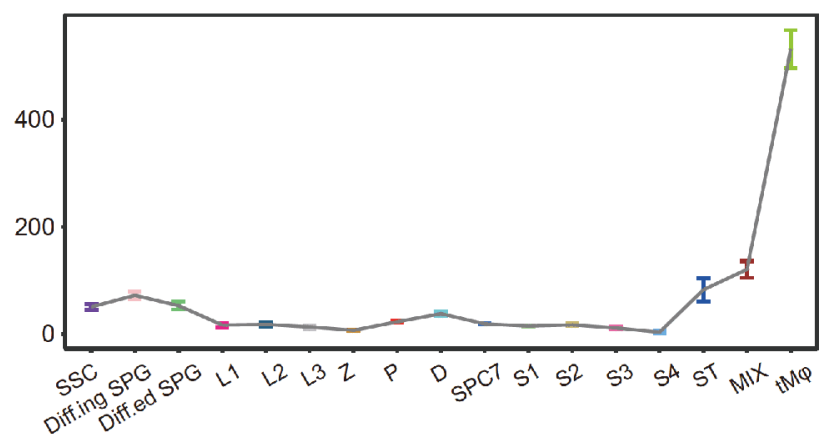

Figure 3 Possible COVID-19 process-related genes expression pattern in normal testis cells. A, The expression levels (TPM) of TMPRSS2 in all cell types. B, Lineplot of ACE2 and TMPRSS2. C, Lineplot of CTSL and CTSB. D, Lineplot of BSG. 

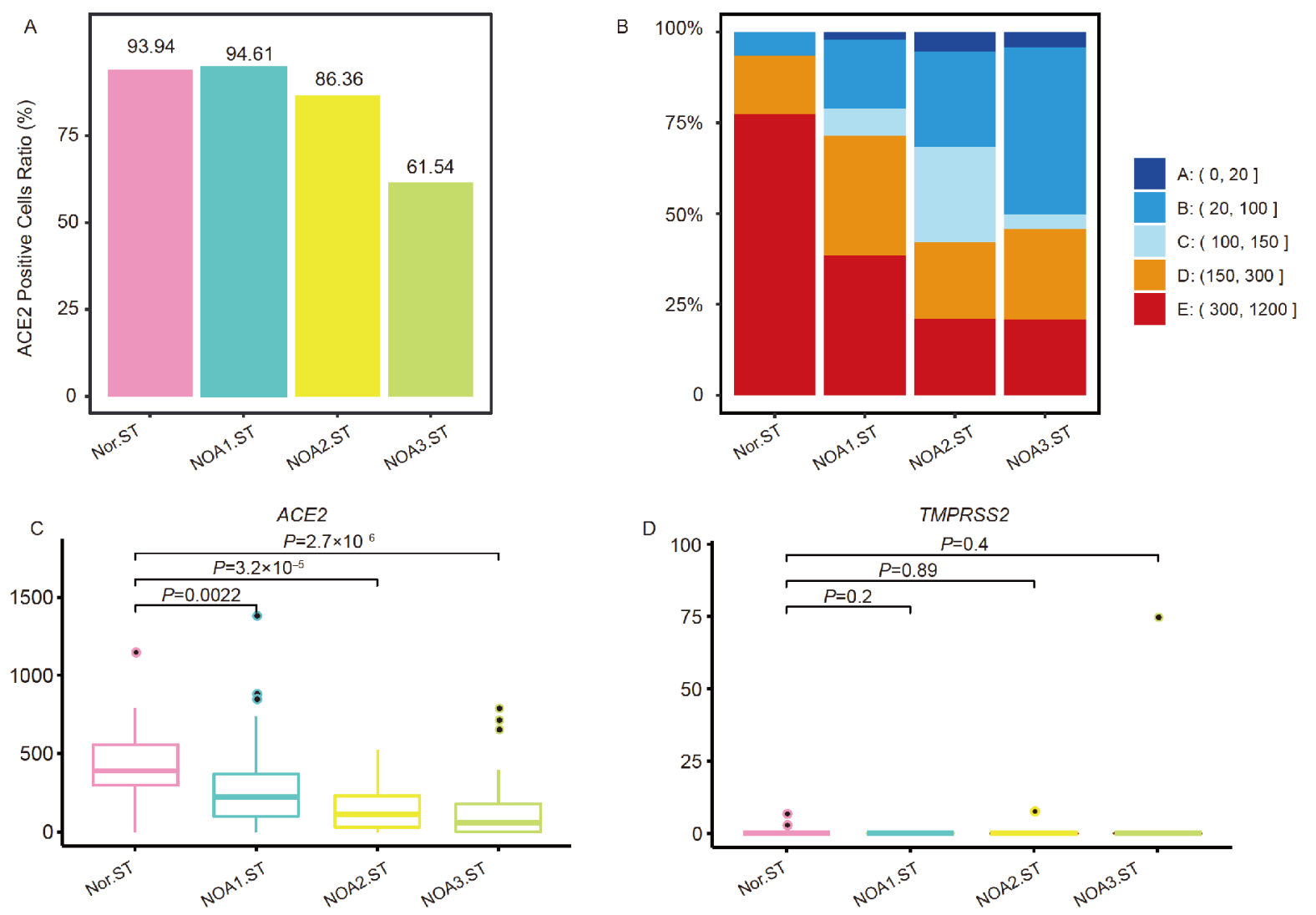

E NOA.ST.D/E vs NOA.ST.F

Up-regulated DEGs
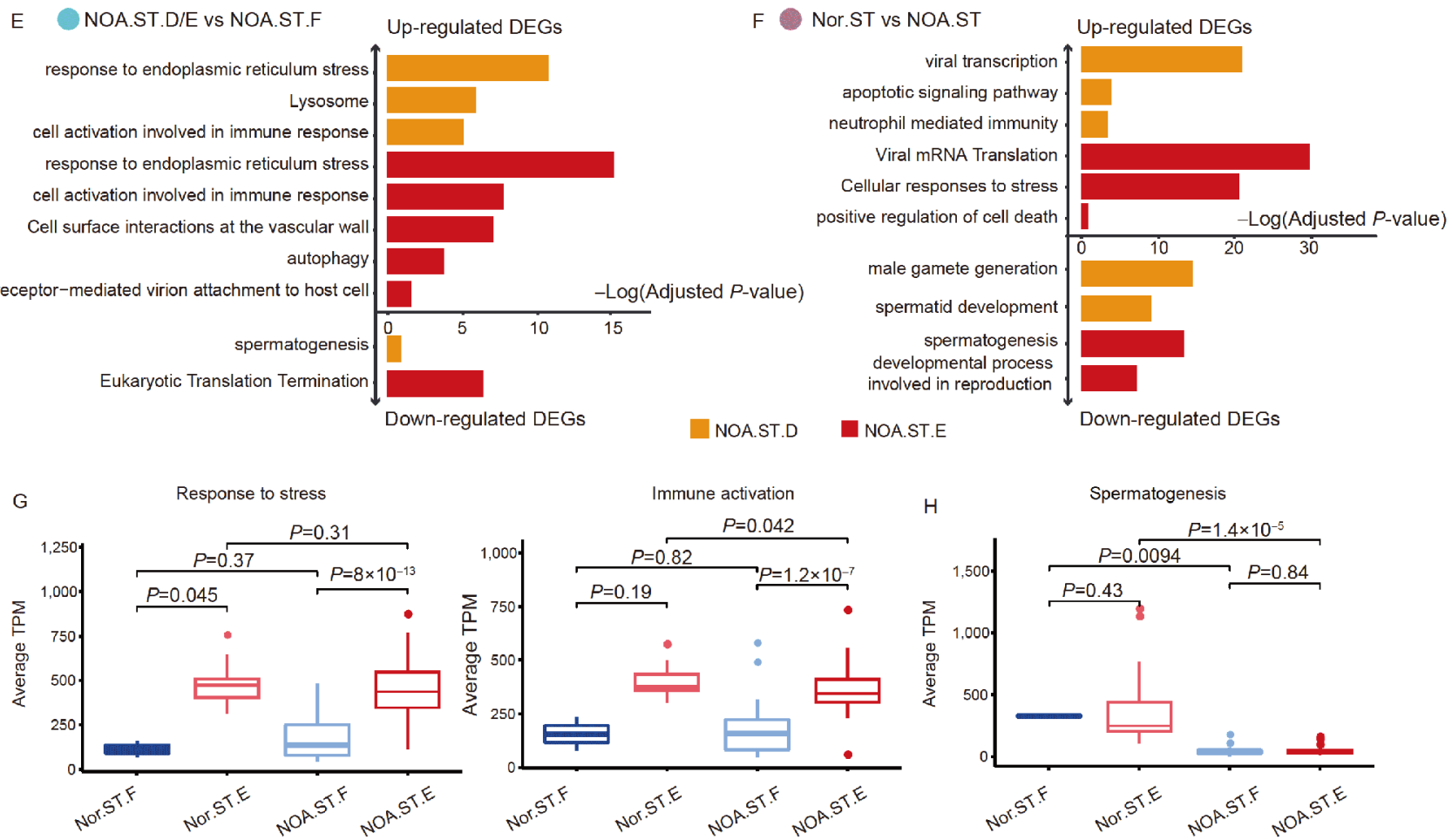

Figure $4 A C E 2$ expression pattern in NOA patients. A, ACE2-positive cells ratio in each Sertoli cell group. Nor.ST refers to Sertoli cells from normal data. NOA.ST refers to Sertoli cells from three non-obstructive azoospermia data. B, Five classes composition distribution in each Sertoli cell group. C, The expression levels of ACE2 in different Sertoli cell groups. D, The expression levels of TMPRSS2 in different Sertoli cell groups. E, Gene ontology terms of DEGs in different classes of Sertoli cells from non-obstructive azoospermia data. DEGs refer to differentially expressed genes. F, Gene ontology terms of DEGs in different classes of Sertoli cells from non-obstructive azoospermia and normal data. DEGs refer to differentially expressed genes. G, The average expression levels of up-regulated gene lists in normal E, F class and NOA E, F class. H, The average expression levels of down-regulated gene list in normal $\mathrm{E}, \mathrm{F}$ class and NOA E, F class. 
cells even when they had a low $A C E 2$ expression level. These data indicate that SARS-CoV-2 may be dangerous to more cell types in human testes including spermatogenic cells and testis somatic cells. Sertoli cells had the highest $A C E 2$ expression level in human male testes, suggesting that Sertoli cells were more susceptible to the effects of the SARS-CoV-2 virus. Based on the variation trend of $A C E 2$, some genes were found with the same variation trend. Whether these genes are related to $A C E 2$ or not deserves further study.

Some possible COVID-19 related genes such as TMPRSS2, BSG, CTSL and CTSB were highly expressed in different cell types. It suggests that if the virus can infect the human testes, there may exist a relationship among several types of testis cells including spermatogenic cells and testis somatic cells. The expression of genes involved in multiple pathways provided more possibilities for virus invasion.

Rennin-angiotensin system (RAS) plays a very important role in human male sperm function (Reis et al., 2010). From our data we found that $A C E 2$ expression levels in three NOA patients all showed significant differences from that of normal Sertoli cells. It suggests that the effect of $A C E 2$ on spermatogenesis is worthy of further study.

Previous studies have shown that a variety of viruses may affect men's testicles such as mumps virus, HIV, HPV and SARS. Some cause inflammation of the testicles through an immune response, while others directly attack the reproductive system. Although the SARS-CoV virus has not been detected in SARS patients' testis tissues (Ding et al., 2004), it has been reported that the spermatogenic cell damage existed in SARS patients (Qi et al., 2007). There is no conclusive evidence that whether SARS-CoV-2 attacks testicular tissue in male patients or not. The expression of $A C E 2$ in testis cells may prompt the possibilities.

A significant increase in serum luteinizing hormone (LH) levels was found in patients with COVID-19, suggesting that COVID-19 may cause male hypogonadism (Ma, 2020). Leydig cells as hormone-secreting cells in the male gonads may have been damaged. From our data we found $A C E 2$, TMPRSS2, BSG, CTSL and CTSB were all expressed in Leydig cells (MIX group), suggesting the possibility that Leydig cells might be influenced by the virus.

In conclusion, our study made a deep analysis to explore the possible COVID-19 related genes expression in different testicular cell types. We also analyzed $A C E 2$ expression in three NOA patients (with and without spermatogenetic cells). Our findings allow better understanding of the potential risks of SARS-CoV-2 infection in different testis cell types and the results may provide more insights into the study of the effects of $A C E 2$ on male infertility. More robust evidence still needs clinical and experimental research.

\section{METHODS}

\section{Single-cell RNA-Seq library preparation and sequencing}

NOA2 and NOA3 donors' testis single cells and scRNA-seq library preparation

The NOA2 and NOA3 donors' testis tissues used in this study were ratified by the Reproductive Study Ethics Committee of Peking University Third Hospital (2017SZ-048). After obtaining the informed consent from the donors undergoing sperm isolation surgery for in vitro fertilization, the testis tissues were collected. The donors' pathology showed the presence of spermatogenic cells but no mature sperms.

The testis tissues were first washed with PBS (plus 10\% FBS) twice, and then $500 \mu \mathrm{L}$ Accutase Cell Detachment Solution (Millipore \#SCR005) was added at $37^{\circ} \mathrm{C}$ and $850 \mathrm{r}$ $\mathrm{min}^{-1}$ for $15 \mathrm{~min}$ to digest tissues completely. After that, we used 50-mm Pre-Separation Filters (Miltenyi Biotec \#130041-407) to filter the cells and centrifuge the cell suspension at $300 \times g, 8 \mathrm{~min}$, and then we used $500 \mu \mathrm{L}$ PBS (plus $10 \%$ FBS) resuspended. Finally, we used the mouth pipette to transfer the single cell to the prepared lysis buffer, and applied the modified STRT-seq protocol (Li et al., 2017) to construct the scRNA-seq library. Sequencing was performed on Illumina HiSeq 4000.

\section{Sequencing and data analysis}

All normal adult human testis samples and NOA1 sample for transcriptional profiles were downloaded from GSE106487 and all PGC transcriptome data were downloaded from GSE86146 in NCBI Gene Expression Omnibus (GEO). NOA2/3 data could be downloaded from HRA000146 in the National Genomics Data Center (https://bigd.big.ac.cn/gsa/). We dealt with all data the same way as mentioned in the literature (Wang et al., 2018).

\section{Raw data processing}

The detailed processing method was as follows. First, we obtained each single cell read 2 data by extracting the specific cell barcode in the first to eighth base of raw read 2 files. Then we added the UMI information which was located in the 9th to 16 th base of raw read 2 files to the corresponding read 1 . The processed read 1 files were then trimmed to remove the template switch oligo (TSO) sequence, polyA tail sequence, adaptor and low-quality reads $(N>10 \%)$. Subsequently, we aligned the clean reads to the hg19 human transcriptome (UCSC) using TopHat (version 2.0.12) (Trapnell et al., 2009). Next, we used HTSeq package to count uniquely mapped reads (Anders et al., 2014). Duplicated transcripts caused by PCR amplification were removed, according to the UMI information. Finally, the transcript copy number of each gene within an individual cell was counted by the distinct UMIs of that gene. For all data 
quality control, we retained the cells with both greater than 2,000 genes and more than 10,000 transcripts.

\section{Nonlinear dimensional reduction (tSNE) and identification of differentially expressed genes}

We used R package Seurat to perform dimension reduction process and identified high variable genes for PCA analysis (Satija et al., 2015). The top PC components were used for the JackStraw analysis and RunTSNE analysis. We used the find_all markers (test.use $=$ "roc") function in Seurat to identify differentially expressed genes (DEGs) and selected DEGs with power $>0.4$ for the following analysis.

\section{Ploting and statistical analysis}

We used R package cummeRbund to identify genes with the same tendency as ACE2 and used ggpubr, ggplot2 to plot boxplots, barplots and lineplots. All gene ontology (GO) analyses were performed in https://metascape.org/ and terms with the FDR-corrected $P$-values below 0.05 were selected. For all normal data, we used wilcox.test with a threshold of 0.05 for significance testing, and for NOA data, we used t. test with a threshold of 0.05 for significance testing.

Compliance and ethics The author(s) declare that they have no conflict of interest.

Acknowledgements This work was funded by Beijing Municipal Science \& Technology Commission (Z191100006619075) and the National Natural Science Foundation of China (81521002 and 81730038).

\section{References}

Anders, S., Pyl, P.T., and Huber, W. (2014). HTSeq-a Python framework to work with high-throughput sequencing data. Bioinformatics $31,166-$ 169.

Backhaus, S., Zakrzewicz, A., Richter, K., Damm, J., Wilker, S., FuchsMoll, G., Küllmar, M., Hecker, A., Manzini, I., Ruppert, C., et al. (2017). Surfactant inhibits ATP-induced release of interleukin-1 $\beta$ via nicotinic acetylcholine receptors. J Lipid Res 58, 1055-1066.

Ding, Y., He, L., Zhang, Q., Huang, Z., Che, X., Hou, J., Wang, H., Shen, H., Qiu, L., Li, Z., et al. (2004). Organ distribution of severe acute respiratory syndrome (SARS) associated coronavirus (SARS-CoV) in SARS patients: implications for pathogenesis and virus transmission pathways. J Pathol 203, 622-630.

Douglas, G.C., O'Bryan, M.K., Hedger, M.P., Lee, D.K.L., Yarski, M.A., Smith, A.I., and Lew, R.A. (2004). The novel angiotensin-converting enzyme (ACE) homolog, ACE2, is selectively expressed by adult Leydig cells of the testis. Endocrinology 145, 4703-4711.

Hoffmann, M., Kleine-Weber, H., Schroeder, S., Krüger, N., Herrler, T., Erichsen, S., Schiergens, T.S., Herrler, G., Wu, N.H., Nitsche, A., et al. (2020). SARS-CoV-2 cell entry depends on ACE2 and TMPRSS2 and is blocked by a clinically proven protease inhibitor. Cell 181, 271-280. e8.

Koguchi, T., Tanikawa, C., Mori, J., Kojima, Y., and Matsuda, K. (2016).
Regulation of myo-inositol biosynthesis by p53-ISYNA1 pathway. Int $\mathrm{J}$ Oncol 48, 2415-2424.

Li, L., Dong, J., Yan, L., Yong, J., Liu, X., Hu, Y., Fan, X., Wu, X., Guo, H., Wang, X., et al. (2017). Single-cell RNA-seq analysis maps development of human germline cells and gonadal niche interactions. Cell Stem Cell 20, 858-873.e4.

Liu, J., Ji, H., Zheng, W., Wu, X., Zhu, J.J., Arnold, A.P., and Sandberg, K. (2010). Sex differences in renal angiotensin converting enzyme 2 (ACE2) activity are 17ß-oestradiol-dependent and sex chromosomeindependent. Biol Sex Dif 1, 6 .

Lu, R., Zhao, X., Li, J., Niu, P., Yang, B., Wu, H., Wang, W., Song, H., Huang, B., Zhu, N., et al. (2020). Genomic characterisation and epidemiology of 2019 novel coronavirus: implications for virus origins and receptor binding. Lancet 395, 565-574.

Ma, L., Xie, W., Li, D., Shi, L., Mao, Y., Xiong, Y., Zhang, Y., and Zhang, M. (2020). Effect of SARS-CoV-2 infection upon male gonadal function: A single center- based study. medRxiv, https://doi.org/10.1101/ 2020.03.21.20037267.

Qi, L., Chi, X., Xu, J., Yang, J., Gong, E., and Gu, J. (2007). Pathological changes of testis tissue in SARS patients. Basic Clin Med.

Qi, S., Huang, M., Teng, H., Lu, Y., Jiang, M., Wang, L., Shi, J., Ma, Q., Gu, G., Xin, Y., et al. (2015). Autoantibodies to chromogranin A are potential diagnostic biomarkers for non-small cell lung cancer. Tumor Biol 36, 9979-9985.

Reis, A.B., Araújo, F.C., Pereira, V.M., Dos Reis, A.M., Santos, R.A., and Reis, F.M. (2010). Angiotensin (1-7) and its receptor Mas are expressed in the human testis: implications for male infertility. J Mol Hist 41, 7580.

Satija, R., Farrell, J.A., Gennert, D., Schier, A.F., and Regev, A. (2015). Spatial reconstruction of single-cell gene expression data. Nat Biotechnol 33, 495-502.

Shen, Q., Xiao, X., Aierken, A., Liao, M., and Hua, J. (2020). The ACE2 expression in Sertoli cells and Germ cells may cause male reproductive disorder after SARS-CoV-2 infection. Preprint, https://doi.org/ 10.31219/osf.io/fs5hd.

Simmons, G., Gosalia, D.N., Rennekamp, A.J., Reeves, J.D., Diamond, S. L., and Bates, P. (2005). Inhibitors of cathepsin L prevent severe acute respiratory syndrome coronavirus entry. Proc Natl Acad Sci USA 102, 11876-11881.

Trapnell, C., Pachter, L., and Salzberg, S.L. (2009). TopHat: discovering splice junctions with RNA-Seq. Bioinformatics 25, 1105-1111.

Wang, K., Chen, W., Zhou, Y.S., Lian, J.Q., Zhang, Z., Du, P., Gong, L., Zhang, Y., Cui, H.Y., Geng, J.J., et al. (2020). SARS-CoV-2 invades host cells via a novel route: CD147-spike protein. bioRxiv, https://doi. org/10.1101/2020.03.14.988345.

Wang, M., Liu, X., Chang, G., Chen, Y., An, G., Yan, L., Gao, S., Xu, Y., Cui, Y., Dong, J., et al. (2018). Single-cell RNA sequencing analysis reveals sequential cell fate transition during human spermatogenesis. Cell Stem Cell 23, 599-614.e4.

Wang, Z., and Xu, X. (2020). scRNA-seq profiling of human testes reveals the presence of the ACE2 receptor, a target for SARS-CoV-2 infection in spermatogonia, Leydig and Sertoli cells. Cells 9, 920-928.

Williamson, C.R., Khurana, S., Nguyen, P., Byrne, C.J., and Tai, T.C. (2017). Comparative analysis of renin-angiotensin system (RAS)related gene expression between hypertensive and normotensive rats. Med Sci Monit Basic Res 23, 20-24.

Zou, X., Chen, K., Zou, J., Han, P., Hao, J., and Han, Z. (2020). Single-cell RNA-seq data analysis on the receptor ACE2 expression reveals the potential risk of different human organs vulnerable to 2019-nCoV infection. Front Med https://doi.org/10.1007/s11684-020-0754-0.

Zuo, X., Xu, W., Xu, M., Tian, R., Moussalli, M.J., Mao, F., Zheng, X., Wang, J., Morris, J.S., Gagea, M., et al. (2017). Metastasis regulation by PPARD expression in cancer cells. JCI Insight 2, e91419. 


\section{SUPPORTING INFORMATION}

Figure S1 Classical testis cell markers expression.

Figure S2 Expression pattern of ACE2 related genes.

Figure S3 The cell number and classical marker expression of Sertoli cells.

The supporting information is available online at http://life.scichina.com and https://link.springer.com. The supporting materials are published as submitted, without typesetting or editing. The responsibility for scientific accuracy and content remains entirely with the authors. 